\title{
Sharp bounds for the number of solutions to simultaneous Pellian equations
}

\author{
by \\ P. G. Walsh (Ottawa)
}

1. Introduction. In [1], Bennett proved that the system of Pell equations

$$
x^{2}-a y^{2}=z^{2}-b y^{2}=1
$$

has at most three solutions in positive integers $x, y, z$. Since then, the result has been improved by Bennett, Cipu, Mignotte and Okazaki in [2], wherein it was shown that this system has at most two solutions in positive integers, which is best possible. The situation is somewhat different for the system of Pell equations

$$
x^{2}-a y^{2}=y^{2}-b z^{2}=1,
$$

which conjecturally has at most one positive integer solution. The best known general bound for the number of positive integer solutions to this system of equations is 2, proved recently by Cipu and Mignotte in [4]. Progress has recently been made by Yuan in [14], in which it was proved that for $a=4 t(t+1)$, the system has at most one solution.

The purpose of this paper is to consider the more general system of Pellian equations

$$
x^{2}-\left(M^{2}-c\right) y^{2}=c, \quad y^{2}-b z^{2}=1, \quad c \in\{ \pm 1, \pm 2, \pm 4\},
$$

where $M$ and $b>1$ are positive integers with $b$ squarefree, and $M^{2}-c$ is a positive nonsquare integer. This is motivated not only by the work of Yuan in [14], but also by a recent paper of Katayama and Levesque [7]. In particular, they considered the case $c=-4$, and proved that under the assumption that the number of distinct prime factors of $b$ is at most 4 , the system (1.1) has at most one solution in positive integers. They also proved that a substantially stronger result follows from the $a b c$ conjecture. We prove here the following. 
TheOREM 1.1. If $M, b, c$ are fixed integers as described above, then the system of Pell equations (1.1) has at most one solution in positive integers $x, y, z$.

There are many examples of equations of the form (1.1) which have a solution in positive integers. For instance, if $c=4$ and $b=M^{2}-1$, then the system has the solution $(x, y, z)=\left(M^{2}-2, M, 1\right)$. Also, if $c=1$ and $b=4 M^{2}-1$, then $(X, Y, Z)=\left(2 M^{2}-1,2 M, 1\right)$ is a solution to (1.1). Consequently, the upper bound of one solution given in Theorem 1.1 is in fact best possible.

2. Preliminary results. Throughout the paper, $c \in\{ \pm 1, \pm 2, \pm 4\}$. Let $M \geq 1$ denote a positive integer, which is odd if $c$ is even, and for which $M^{2}-c$ is a positive nonsquare integer. Let

$$
\alpha=\frac{M+\sqrt{M^{2}-c}}{\sqrt{|c|}},
$$

and for $i \geq 1$, define sequences $\left\{V_{i}\right\}$ and $\left\{W_{i}\right\}$ by

$$
\alpha^{i}=\frac{V_{i}+W_{i} \sqrt{M^{2}-c}}{\sqrt{|c|}} .
$$

Also, for $b>1$ and squarefree, let $\beta=T+U \sqrt{b}$ denote the smallest unit greater than 1 in $\mathbb{Z}[\sqrt{b}]$ which is of norm 1 , and for $j \geq 1$, let

$$
\beta^{j}=T_{j}+U_{j} \sqrt{b}
$$

The following is similar to Lemma 2.1 of [14]. The proof of these statements follows from the binomial theorem.

LEMMA 2.1.

(i) If $|c|=4$, then $V_{i}$ and $W_{i}$ are both even if 3 divides $i$, and both odd otherwise.

(ii) $W_{i}$ divides $W_{j}$ if and only if $i$ divides $j$.

(iii) $V_{i}$ divides $V_{j}$ if and only if $i / j$ is an odd integer.

(iv) If $d=\operatorname{gcd}(i, j)$, then $\operatorname{gcd}\left(W_{i}, W_{j}\right)=W_{d}$.

(v) If $d=\operatorname{gcd}(i, j)$, then $\operatorname{gcd}\left(V_{i}, V_{j}\right)=V_{d}$ if $i / d$ and $j / d$ are odd integers, and 1 otherwise.

(vi) $W_{2 i}=V_{i} W_{i}$ if $c$ is even, and $W_{2 i}=2 V_{i} W_{i}$ if $c$ is odd.

The following is similar to Lemmas 2.2 and 2.3 from [14]. The proof follows from direct computation, and taking into consideration the facts in the previous lemma.

LEMma 2.2. Let $k_{0}, k_{1}, k_{2}$ and $q$ be positive integers with $k_{2}=2 q k_{1} \pm k_{0}$ and $0 \leq k_{0} \leq k_{1}$. Then 
(i) If $|c| \neq 4$, or if $|c|=4$ and $V_{k_{1}}$ is odd, then $V_{k_{2}} \equiv \pm V_{k_{0}}\left(\bmod V_{k_{1}}\right)$.

(ii) If $|c|=4$ and $V_{k_{1}}$ is even, then $V_{k_{2}} \equiv \pm V_{k_{0}}\left(\bmod V_{k_{1}} / 2\right)$.

(iii) If $|c| \neq 4$, or if $|c|=4$ and $W_{k_{1}}$ is odd, then $W_{k_{2}} \equiv \pm W_{k_{0}}$ $\left(\bmod W_{k_{1}}\right)$.

(iv) If $|c|=4$ and $W_{k_{1}}$ is even, then $W_{k_{2}} \equiv \pm W_{k_{0}}\left(\bmod W_{k_{1}} / 2\right)$.

The following is similar to Lemma 2.4 of [14], and is the vital observation underlying the method of this paper. We will provide the details of the proof for $c=-4$, as this is the case that presents the most difficulty.

Assume that (1.1) has a solution in positive integers. Let $\left(x_{0}, y_{0}, z_{0}\right)$ denote the solution to (1.1) with $z_{0}$ minimal. Let $k_{0}, l_{0}$ denote the positive integers for which $y_{0}=W_{k_{0}}$ and $z_{0}=U_{l_{0}}$. Also, $(x, y, z)$ will denote a different solution to (1.1), and $k$ and $l$ will denote positive integers for which $y=W_{k}$, and $z=U_{l}$.

Lemma 2.3. Assume that $(M, b)$ is not one of $\{(1,2),(1,3),(1,15)\}$. Then $y_{0}\left|y, z_{0}\right| z$, and $k / k_{0}$ and $l / l_{0}$ are odd integers.

Proof. Assume that $k / k_{0}$ and $l / l_{0}$ are not odd integers. Then there are integers $s, q, t, q_{1}$ for which $0 \leq s<k_{0}, k=2 q k_{0} \pm s, 0 \leq t<l_{0}, q_{1}$, and $l=2 q_{1} l_{0} \pm t$. We find from Lemma 2.2 that

$$
y=W_{k} \equiv \pm W_{s}\left(\bmod W_{k_{0}} / 2^{\delta}\right) \equiv \pm W_{s}\left(\bmod y_{0} / 2^{\delta}\right),
$$

where $\delta=0$ if $y_{0}$ is even, and $\delta=1$ if $y$ is odd. Similarly, by Lemma 2.3 in [14],

$$
y=T_{l} \equiv \pm T_{t}\left(\bmod T_{l_{0}}\right) \equiv \pm T_{t}\left(\bmod y_{0}\right) .
$$

Therefore,

$$
W_{s} \equiv \pm T_{t}\left(\bmod y_{0} / 2^{\delta}\right)
$$

Since $k$ is odd, $s$ is odd. Since $k_{0}$ is odd and larger than $s$, it follows that $k_{0} \geq s+2$. Therefore, by the assumption that $M>1$ is odd, and a basic estimate for the growth rate of the sequence $\left\{W_{i}\right\}$, it follows that

$$
W_{s}<(1 / 4) W_{s+2} \leq(1 / 4) W_{k_{0}}=(1 / 4) y_{0} .
$$

Also, because $b$ is not one of 2,3 or 15 , the growth rate of the sequence $\left\{T_{i}\right\}$ implies that

$$
T_{t}<(1 / 4) T_{t+1} \leq(1 / 4) T_{l_{0}}=(1 / 4) y_{0} .
$$

Because of these estimates, we see that the minus sign in (2.3) is not possible. Therefore, $W_{s} \equiv T_{t}\left(\bmod y_{0} / 2^{\delta}\right)$ must hold. But in this case, the estimates imply that $W_{s}=T_{t}$, which contradicts the fact that $\left(x_{0}, y_{0}, z_{0}\right)$ is the smallest solution to (1.1). It follows that at least one of $k / k_{0}$ or $l / l_{0}$ is an odd integer. By the lemma above, $k / k_{0}$ is an odd integer if and only if $y_{0}$ divides $y$, and this occurs if and only if $l / l_{0}$ is an odd integer. The lemma follows. 
The method also uses, in a fundamental way, the results of Voutier [12] and Bilu, Hanrot and Voutier [3] on the existence of primitive divisors in Lucas sequences. Combining the results of those papers yields the following result. We first remind the reader of the notion of a primitive prime factor.

Definition. Let $\left\{W_{i}\right\}$ be the sequence of integers in (2.2). We say that $W_{i}$ has a primitive prime factor if there is a prime factor of $W_{i}$ which does not divide $W_{j}$ for all $1 \leq j \leq i-1$.

The rank of apparition $r(m)$ of a positive integer $m>1$ in the sequence $\left\{W_{i}\right\}$ is the smallest positive integer $i$ for which $m$ divides $W_{i}$. Thus we see that if $m$ is prime, then it is a primitive prime factor of $W_{r(m)}$. The main point of these definitions is the well known result that $m>1$ divides $W_{k}$ if and only if $r(m)$ divides $k$.

Lemma 2.4. Let $\alpha$ be as in (2.1). Then for $i>1, W_{i}$ has a primitive prime factor except only if $\alpha=(1+\sqrt{5}) / 2$ and $i \in\{2,6,12\}$.

We finish off this series of lemmata by a result which combines results of Ljunggren [8], Cohn [6], and the author [13]. It essentially solves the problem of determining all instances when the product of two distinct elements in any sequence $\left\{W_{i}\right\}$ is a square.

Lemma 2.5. Let $C=1$ if $|c|=1$ or $|c|=2$, and $C=4$ if $|c|=4$. For any positive integer $A$, there is at most one positive integer solution $X, Y$ to

$$
X^{2}-\left(M^{2}-c\right) Y^{2}=C
$$

with $Y=A \cdot u^{2}$, for some integer $u$, except only in the following cases.

(i) $c=1, A=1, M=2 m^{2}$, in which case $Y \in\left\{1,(2 m)^{2}\right\}$.

(ii) $c=1, A=1, M=169$, in which case $Y \in\left\{1,(6214)^{2}\right\}$.

(iii) $c=2, A=m_{1}$ with $M=m_{1} u^{2}$, and $M^{2}-1=2 m^{2}$, in which case $Y \in\left\{M,(2 m)^{2} M\right\}$.

(iv) $c=-2, A=m_{1}$ with $M=m_{1} u^{2}$, and $M^{2}+1=2 m^{2}$, in which case $Y \in\left\{1,(2 m)^{2} M\right\}$.

(v) $c=4, M=1, A=1$, in which case $Y \in\{1,144\}, M=1, A=2$, in which case $Y \in\{2,8\}$, or $M=m^{2}>1, A=1$, in which case $Y \in\left\{1, m^{2}\right\}$.

Proof. We prove this by considering each value of $c$ separately. We retain the notation from (2.4).

CASE 1: $c=1$. Assume that there are two positive indices $k<l$ for which $W_{k}=A u^{2}$ and $W_{l}=A v^{2}$ for some positive integers $u$ and $v$. By a recent improvement to Ljunggren's theorem on the equation $X^{2}-D Y^{2}=1$ in [11], either $A^{2}\left(M^{2}-1\right)=1785, A^{2}\left(M^{2}-1\right)=16 \cdot 1785$, or else $V_{k}+A u^{2} \sqrt{M^{2}-1}$ is the smallest unit greater than 1 in $\mathbb{Z}\left[\sqrt{M^{2}-1}\right]$ which is of norm 1 , and 
$V_{l}+A v^{2} \sqrt{M^{2}-1}$ is its square. The equation $A^{2}\left(M^{2}-1\right)=1785$ is not solvable, while the equation $A^{2}\left(M^{2}-1\right)=16 \cdot 1785$ leads to case (ii) in the statement of the lemma. Finally, if the third possibility occurs, then $V_{k}+A u^{2} \sqrt{M^{2}-1}=M+\sqrt{M^{2}-1}$ and $V_{l}+A v^{2} \sqrt{M^{2}-1}=2 M^{2}-1+$ $2 M \sqrt{M^{2}-1}$. Therefore, $A=1, u=1$, and $v^{2}=2 M$, which implies that $M=2 m^{2}$ for some integer $m$, resulting in (i) in the statement of the lemma.

CASE 2: $c=-1$. By the same argument as in the previous case, but appealing directly to Ljunggren's theorem in [8] (or see Theorem 9 on p. 270 in [9]), it follows that $V_{k}+W_{k} \sqrt{M^{2}+1}$ is the fundamental unit in $\mathbb{Z}\left[\sqrt{M^{2}+1}\right]$. This is not possible since the fundamental unit in that ring has norm -1 .

CASE 3: $c= \pm 2$. The minimal unit in $\mathbb{Z}\left[\sqrt{M^{2} \pm 2}\right]$ is $M^{2} \pm 1+M \sqrt{M^{2} \pm 2}$, and so the argument given to prove Case 2 shows $A u^{2}=M$ and $A v^{2}=$ $2\left(M^{2} \pm 1\right) M$. This forces $M^{2} \pm 1=2 m^{2}$ for some positive integer $m$.

CASE 4: $c=4$. Assume that $M^{2}-4>5$, since the case $M=1$ is not possible and $M=3$ was dealt with by Ribenboim in [10]. Assume first that the equation $X^{2}-A^{2}\left(M^{2}-4\right) Y^{2}=4$ is solvable in odd integers $X, Y$ and let

$$
\alpha_{A}=\frac{v_{1}+w_{1} \sqrt{A^{2}\left(M^{2}-4\right)}}{2}
$$

denote its minimal solution. For $i \geq 1$, we let

$$
\alpha_{A}^{i}=\frac{v_{i}+w_{i} \sqrt{A^{2}\left(M^{2}-4\right)}}{2} .
$$

Thus there are integers $k_{1}$ and $l_{1}$ for which $w_{k_{1}}=u^{2}$ and $w_{l_{1}}=v^{2}$. By Theorem 3 of [6] applied to $d=A^{2}\left(M^{2}-4\right)$, we find that $k_{1}=1, l_{1}=2$ and furthermore that $v_{1}$ is a square. But $v_{1}=V_{k}$, and so applying Theorem 1 of [6], we see that $k=1$. Therefore, $M=m^{2}$ for some integer $m, A=1$, and $W_{k}=1, W_{l}=m^{2}$.

Assume now that the equation $X^{2}-A^{2}\left(M^{2}-4\right) Y^{2}=4$ is not solvable in odd integers $X, Y$. Let $v_{k}=V_{3 k} / 2$ and $w_{k}=W_{3 k} / 2$, so that $(X, Y)=$ $\left(v_{k}, w_{k}\right)$ constitute all solutions to $X^{2}-\left(M^{2}-4\right) Y^{2}=1$. By assumption, there are indices $k$ and $l$ for which $w_{k}=\left(A u^{2}\right) / 2$ and $w_{l}=\left(A v^{2}\right) / 2$, and it follows from Theorem 1 in [13], with $D=A^{2}\left(M^{2}-4\right)$, that $k=1$ and $l=2$. This in turn implies that $v_{k}=2 V_{3 k}$ is a square, which is not possible by Theorem 2 of [6].

CASE 5: $c=-4$. Again we may assume that $M^{2}+4>5$ by the result of Ribenboim [10]. Assume first that the equation $X^{2}-A^{2}\left(M^{2}+4\right) Y^{2}=4$ is solvable in odd integers $X, Y$ and let

$$
\alpha_{A}=\frac{v_{1}+w_{1} \sqrt{A^{2}\left(M^{2}+4\right)}}{2}
$$


denote its minimal solution. For $i \geq 1$, we let

$$
\alpha_{A}^{i}=\frac{v_{i}+w_{i} \sqrt{A^{2}\left(M^{2}+4\right)}}{2} .
$$

Thus there are integers $k_{1}$ and $l_{1}$ for which $w_{k_{1}}=u^{2}$ and $w_{l_{1}}=v^{2}$. By Theorem 3 of [5], this forces $k_{1}=1$ and $v_{1}$ to be a square. But $v_{1}=V_{k}$, and so by Theorem 1 of [5], either $k=1$, or $k=3$ and $A^{2}\left(M^{2}+4\right)=13$. The latter is not possible since $k$ is evidently even.

Assume now that the equation $X^{2}-A^{2}\left(M^{2}+4\right) Y^{2}=4$ is not solvable in odd integers $X, Y$. Let $v_{k}=V_{3 k} / 2$ and $w_{k}=W_{3 k} / 2$, so that $(X, Y)=$ $\left(v_{k}, w_{k}\right)$ constitute all solutions to $X^{2}-\left(M^{2}+4\right) Y^{2}=1$. By assumption, there are indices $k$ and $l$ for which $w_{k}=\left(A u^{2}\right) / 2$ and $w_{l}=\left(A v^{2}\right) / 2$, and it follows from Theorem 1 in [13], with $D=A^{2}\left(M^{2}+4\right)$, that $k=1$ and $l=2$. This in turn implies that $v_{k}=2 V_{3 k}$ is a square, which is not possible by Theorem 2 of $[6]$.

3. Proof of Theorem 1.1. Assume that (1.1) is solvable in positive integers, and let $\left(x_{0}, y_{0}, z_{0}\right)$ denote the smallest positive integer solution to (1.1). Let $\left(x_{1}, y_{1}, z_{1}\right)$ denote a larger solution (specifically meaning that $z_{0}<z_{1}$ ). Let $k_{0}, l_{0}, k_{1}, l_{1}$ be the corresponding powers of $\alpha$ and $\beta$, as defined at the start of Section 2.

We will first consider the case $c=1$ in detail. The proof for the other cases will be given with less detail in order to keep the presentation at a reasonable length.

There are two distinct cases to consider depending on the parity of $y_{0}$. Assume first that $y_{0}$ is odd. It follows from Lemma 2.3 that $y_{1}$ is also odd.

Since $y_{0}$ is odd, $x_{0}+y_{0} \sqrt{M^{2}-1}$ is an odd power of $M+\sqrt{M^{2}-1}$, and so $M$ divides $x_{0}$. Subtracting the second equation in (1.1) from the first yields

$$
M^{2} y_{0}^{2}-x_{0}^{2}=b z_{0}^{2} .
$$

Since $M$ divides $x_{0}$ and $b$ is squarefree, it follows that $M$ also divides $z_{0}$. Put $X_{0}=x_{0} / M$ and $Z_{0}=z_{0} / M$, then (3.1) becomes

$$
y_{0}^{2}-X_{0}^{2}=b Z_{0}^{2} \text {. }
$$

We note that $V_{2 i+1} / V_{1}$ is an odd integer for all $i \geq 0$, which shows that $X_{0}$ is odd, and hence also that $Z_{0}$ is even. Therefore, there are positive integers $A_{0}, B_{0}, u_{0}, v_{0}$, with $b=A_{0} B_{0}$ and $Z_{0}=2 u_{0} v_{0}$, for which

$$
y_{0}+X_{0}=2 A_{0} u_{0}^{2}, \quad y_{0}-X_{0}=2 B_{0} v_{0}^{2} .
$$

Since $b$ is squarefree, we note that $\operatorname{gcd}\left(A_{0}, B_{0}\right)=1$. Also, $\operatorname{since} \operatorname{gcd}\left(x_{0}, y_{0}\right)=1$, it follows that $\operatorname{gcd}\left(y_{0}+X_{0}, y_{0}-X_{0}\right)=2$, which yields $\operatorname{gcd}\left(A_{0} u_{0}^{2}, B_{0} v_{0}^{2}\right)=1$. 
From (3.2) we see that $y_{0}=A_{0} u_{0}^{2}+B_{0} v_{0}^{2}$, and so substituting $y_{0}$ and $z_{0}=2 M u_{0} v_{0}$ into the second equation in (1.1), and then simplifying, gives

$$
\left(A_{0} u_{0}^{2}+\left(1-2 M^{2}\right) B_{0} v_{0}^{2}\right)^{2}-\left(M^{2}-1\right)\left(2 M B_{0} v_{0}^{2}\right)^{2}=1 .
$$

The symmetry of this equation shows that it can also be written as

$$
\left(B_{0} v_{0}^{2}+\left(1-2 M^{2}\right) A_{0} u_{0}^{2}\right)^{2}-\left(M^{2}-1\right)\left(2 M A_{0} u_{0}^{2}\right)^{2}=1 .
$$

Therefore, there is a positive integer $i_{0}$ for which $W_{i_{0}}=2 M B_{0} v_{0}^{2}$. Since $M$ divides $W_{i_{0}}$, it follows that $i_{0}$ is even. Similarly, there is also an even positive integer $j_{0}$ for which $W_{j_{0}}=M A_{0} u_{0}^{2}$. Similarly, the second solution to (1.1), namely $\left(x_{1}, y_{1}, z_{1}\right)$, shows the existence of positive integers $A_{1}, B_{1}, u_{1}, v_{1}, i_{1}, j_{1}$, with $i_{1}$ and $j_{1}$ even, for which $b=A_{1} B_{1}, z_{1}=2 M u_{1} v_{1}$, $W_{i_{1}}=2 M B_{1} v_{1}^{2}$, and $W_{j_{1}}=2 M A_{1} u_{1}^{2}$. We remark that, as with the previous solution, $\operatorname{gcd}\left(A_{1}, B_{1}\right)=1$ and $\operatorname{gcd}\left(A_{1} u_{1}^{2}, B_{1} v_{1}^{2}\right)=1$. These remarks concerning greatest common divisors imply by Lemma 2.1 that $\operatorname{gcd}\left(i_{0}, j_{0}\right)=$ $\operatorname{gcd}\left(i_{1}, j_{1}\right)=2$, and that $\operatorname{gcd}\left(W_{i_{0}}, W_{j_{0}}\right)=\operatorname{gcd}\left(W_{i_{1}}, W_{j_{1}}\right)=W_{2}=2 M$.

By Lemma 2.4, since $i_{0}>1$ and $j_{0}>1, W_{i_{0}}$ and $W_{j_{0}}$ each have a primitive prime factor, which will be denoted as $p$ and $q$ respectively. By Lemma $2.3, z_{0}$ divides $z_{1}$, and since $A_{0} B_{0}=A_{1} B_{1}$, it follows that $p$ must divide one of $W_{i_{1}}$ or $W_{j_{1}}$. Therefore, by the remarks concerning the rank of apparition in Section 2, $i_{0}$ divides one of $i_{1}$ or $j_{1}$. Similarly, $j_{0}$ divides one of $i_{1}$ or $j_{1}$.

Assume first that both $i_{0}$ and $j_{0}$ divide $i_{1}$. It follows that $W_{i_{0}}$ and $W_{j_{0}}$ divide $W_{i_{1}}$, and hence that $W_{i_{0}} / M$ and $W_{j_{0}} / M$ divide $W_{i_{1}} / M$. We claim that this implies that $A_{1}=1$. If $p_{1}$ is a prime dividing $A_{1}$, then $p_{1}$ divides one of $A_{0}$ or $B_{0}$, and hence it divides at least one of $W_{i_{0}} / M$ or $W_{j_{0}} / M$. Therefore, $p_{1}$ divides $W_{i_{1}} / M$. But since $A_{1}$ divides $W_{j_{1}} / M$, it follows that $p_{1}$ divides $\operatorname{gcd}\left(W_{i_{1}} / M, W_{j_{1}} / M\right)$, which is equal to 2 by the remarks above. Thus, $A_{1}=1$ or $A_{1}=2$. If $A_{1}=2$, then the equation $W_{i}=4 M X^{2}$ would be solvable, and since $W_{2}=2 M \cdot 1^{2}$, Theorem 1 of [13] applied to $D=M^{2}\left(M^{2}-1\right)$ implies that $M=1$, which is not possible. Therefore, $A_{1}=1$ as claimed. Since $W_{j_{1}}=2 M A_{1} u_{1}^{2}$, it follows that $W_{j_{1}}=2 M u_{1}^{2}$, and Lemma 2.5 implies that $j_{1}=2$ and $u_{1}=1$. Therefore, from the construction of the integers $A_{1}, u_{1}$ from $y_{1}, X_{1}$, it follows that $y_{1} \pm X_{1}=2$, and since $y_{1} \geq 2$, it follows that $y_{1}-X_{1}=2$. This implies that $M y_{1}-x_{1}=2 M$, from which it follows that $x_{1}=M\left(y_{1}-2\right)$. Substituting this for $x$ in the first equation in (1.1) and simplifying gives $y_{1}=4 M^{2}-1=W_{3}$. Since $y_{0}>1$ and odd, it follows that $y_{0}=W_{k_{0}} \geq W_{3}=y_{1}$, contradicting the fact that $y_{0}<y_{1}$.

We can now assume, without loss of generality, that $i_{0}$ divides $i_{1}$ and $j_{0}$ divides $j_{1}$. Then $W_{i_{0}}$ divides $W_{i_{1}}$ and $W_{j_{0}}$ divides $W_{j_{1}}$, which implies that $B_{0} u_{0}^{2}$ divides $B_{1} u_{1}^{2}$ and $A_{0} v_{0}^{2}$ divides $A_{1} v_{1}^{2}$. Now suppose that $p$ is a 
prime dividing $\operatorname{gcd}\left(B_{0}, A_{1}\right)$; then $p$ divides $\operatorname{gcd}\left(W_{i_{1}} /(2 M), W_{j_{1}} /(2 M)\right)=1$, a contradiction. Therefore, $B_{0}$ divides $B_{1}$, and a similar argument shows that $A_{0}$ divides $A_{1}$. Since $A_{0} B_{0}=A_{1} B_{1}$, the only way this can occur is if $A_{0}=A_{1}$ and $B_{0}=B_{1}$. By Lemma 2.5 , this forces $A_{0}=B_{0}=1$, and so $b=1$, which is not possible.

Now assume that $y_{0}$ is even. Then $y_{1}$ is also even, and all of $x_{0}, x_{1}, z_{0}, z_{1}$ are odd. In this case, the factors of the left side in (3.1) are coprime, and so there are odd positive integers $A_{0}, B_{0}, A_{1}, B_{1}, u_{0}, v_{0}, u_{1}, v_{1}$, with $b=A_{0} B_{0}=$ $A_{1} B_{1}, z_{0}=u_{0} v_{0}, z_{1}=u_{1} v_{1}$, for which

$$
M y_{i}-x_{i}=A_{i} u_{i}^{2}, \quad M y_{i}+x_{i}=B_{i} v_{i}^{2} \quad(i=0,1),
$$

and for $i=0,1, \operatorname{gcd}\left(A_{i}, B_{i}\right)=\operatorname{gcd}\left(u_{i}, v_{i}\right)=1$. This gives

$$
y_{i}=\left(A_{i} u_{i}^{2}+B_{i} v_{i}^{2}\right) /(2 M) \quad(i=0,1),
$$

and substituting this and $z_{i}=u_{i} v_{i}$ into the second equation in (1.1) and simplifying results in the equation

$$
\left(\frac{A_{i} u_{i}^{2}+\left(1-2 M^{2}\right) B_{i} v_{i}^{2}}{2 M}\right)^{2}-\left(M^{2}-1\right) B_{i}^{2} v_{i}^{4}=1 \quad(i=0,1) .
$$

By symmetry, one also obtains an identical equation, but with the $A_{i}$ (resp. $u_{i}$ ) and $B_{i}$ (resp. $v_{i}$ ) interchanged.

Therefore, there are odd positive indices $i_{0}, j_{0}, i_{1}, j_{1}$ for which

$$
W_{i_{0}}=B_{0} v_{0}^{2}, \quad W_{j_{0}}=A_{0} u_{0}^{2}, \quad W_{i_{1}}=B_{1} v_{1}^{2}, \quad W_{j_{1}}=A_{1} u_{1}^{2} .
$$

As argued in the previous case, Lemmas 2.3 and 2.4 imply that both $W_{i_{0}}$ and $W_{j_{0}}$ divide one of $W_{i_{1}}$ and $W_{j_{1}}$. If they both divide say $W_{i_{1}}$, it follows, as argued in the previous case, that $A_{1}=1$. Therefore, $W_{j_{1}}=u_{1}^{2}$ is a square, and by Lemma 2.5 , it follows that $W_{j_{1}}=1$, which in turn implies by (3.4) that $M y_{1}-x_{1}=1$. Substituting this quantity into the first equation in (1.1) and simplifying shows that $y_{1}=2 M=W_{2}$. Since $y_{0}$ is even, we already knew that $y_{0} \geq 2 M$, and so this contradicts the fact that $y_{0}<y_{1}$.

We now consider the case $c=-1$. In this case, $y_{0}$ and $y_{1}$ must be odd, and $X_{0}=x_{0} / M$ and $X_{1}=x_{1} / M$ are odd integers. Adding the two equations in (1.1) and dividing by $M$ gives

$$
X_{i}^{2}-y_{i}^{2}=b Z_{i}^{2} \quad(i=0,1),
$$

where $Z_{0}=z_{0} / M, Z_{1}=z_{1} / M$ are even integers. It follows that there exist positive integers $A_{0}, B_{0}, A_{1}, B_{1}$ and $u_{0}, u_{1}, v_{0}, v_{1}$ for which $b=A_{0} B_{0}=$ $A_{1} B_{1}, Z_{0}=2 u_{0} v_{0}, Z_{1}=2 u_{1} v_{1}$, and

$$
X_{i}-y_{i}=2 A_{i} u_{i}^{2}, \quad X_{i}+y_{i}=2 B_{i} v_{i}^{2} \quad(i=0,1) .
$$

Solving for $y_{i}$, substituting $y_{i}$ and $z_{i}$ in the second equation in (1.1), and then simplifying gives

$$
\left(A_{i} u_{i}^{2}+\left(1+2 M^{2}\right) B_{i} v_{i}^{2}\right)^{2}-\left(M^{2}+1\right)\left(2 M B_{i} v_{i}^{2}\right)^{2}=1 \quad(i=0,1)
$$


and

$$
\left(B_{i} v_{i}^{2}+\left(1+2 M^{2}\right) A_{i} u_{i}^{2}\right)^{2}-\left(M^{2}+1\right)\left(2 M A_{i} u_{i}^{2}\right)^{2}=1 \quad(i=0,1) .
$$

The rest of the proof follows exactly as in the case $c=1$ with $y_{0}$ odd, and so we forego the details.

Now assume that $c=2$, in which case $M$ is assumed to be odd. It follows that $y_{0}$ and $y_{1}$ are odd, $z_{0}$ and $z_{1}$ are even, and both $X_{0}=x_{0} / M, X_{1}=x_{1} / M$ are odd integers. Subtracting twice the second equation from the first in (1.1) and dividing by $M$, gives

$$
y_{i}^{2}-X_{i}^{2}=2 b Z_{i}^{2} \quad(i=0,1),
$$

where $Z_{0}=z_{0} / M, Z_{1}=z_{1} / M$. It follows that there exist positive integers $A_{0}, B_{0}, A_{1}, B_{1}, u_{0}, u_{1}, v_{0}, v_{1}$ for which $2 b=A_{0} B_{0}=A_{1} B_{1}, Z_{0}=2 u_{0} v_{0}, Z_{1}=$ $2 u_{1} v_{1}$, and

$$
y_{i}-X_{i}=2 A_{i} u_{i}^{2}, \quad y_{i}+X_{i}=2 B_{i} v_{i}^{2} \quad(i=0,1) .
$$

Solving for $y_{i}$, substituting $y_{i}$ and $z_{i}$ in the second equation in (1.1), and then simplifying gives

$$
\left(A_{i} u_{i}^{2}+\left(1-M^{2}\right) B_{i} v_{i}^{2}\right)^{2}-\left(M^{2}-2\right)\left(2 M B_{i} v_{i}^{2}\right)^{2}=1 \quad(i=0,1),
$$

and by symmetry

$$
\left(B_{i} v_{i}^{2}+\left(1-M^{2}\right) A_{i} u_{i}^{2}\right)^{2}-\left(M^{2}-2\right)\left(2 M A_{i} u_{i}^{2}\right)^{2}=1 \quad(i=0,1) .
$$

The rest of the proof follows exactly as in the case $c=1$ with $y_{0}$ odd, and so we forego the details.

Now assume that $c=-2$, in which case $M$ is assumed to be odd. It follows that $y_{0}$ and $y_{1}$ are odd, $z_{0}$ and $z_{1}$ are even, and both $X_{0}=x_{0} / M, X_{1}=$ $x_{1} / M$ are odd integers. Adding twice the second equation to the first in (1.1) and dividing by $M$ gives

$$
X_{i}^{2}-y_{i}^{2}=2 b Z_{i}^{2} \quad(i=0,1) .
$$

It follows that there exist positive integers $A_{0}, B_{0}, A_{1}, B_{1}, u_{0}, u_{1}, v_{0}, v_{1}$ for which $2 b=A_{0} B_{0}=A_{1} B_{1}, Z_{0}=2 u_{0} v_{0}, Z_{1}=2 u_{1} v_{1}$, and

$$
X_{i}-y_{i}=2 A_{i} u_{i}^{2}, \quad X_{i}+y_{i}=2 B_{i} v_{i}^{2} \quad(i=0,1) .
$$

Solving for $y_{i}$, substituting $y_{i}$ and $z_{i}$ in the second equation in (1.1), and then simplifying gives

$$
\left(A_{i} u_{i}^{2}-\left(1+M^{2}\right) B_{i} v_{i}^{2}\right)^{2}-\left(M^{2}+2\right)\left(2 M B_{i} v_{i}^{2}\right)^{2}=1 \quad(i=0,1),
$$

and by symmetry

$$
\left(B_{i} v_{i}^{2}-\left(1+M^{2}\right) A_{i} u_{i}^{2}\right)^{2}-\left(M^{2}+2\right)\left(2 M A_{i} u_{i}^{2}\right)^{2}=1 \quad(i=0,1) .
$$

The rest of the proof follows exactly as in the case $c=1$ with $y_{0}$ odd.

Now we consider the case $c=4$. Let $k_{0}$ and $k_{1}$ be indices for which $y_{0}=W_{k_{0}}$ and $y_{1}=W_{k_{1}}$. By Lemma $2.3, k_{0}$ and $k_{1}$ have the same parity. 
Assume first that they are both odd. In this case $M$ divides $x_{0}$ and $x_{1}$. Multiplying the second equation in (1.1) by 4, subtracting it from the first equation, and dividing the result by $M$ gives

$$
y_{i}^{2}-X_{i}=4 b Z_{i} \quad(i=0,1),
$$

where as before, $X_{0}=x_{0} / M, X_{1}=x_{1} / M$ and $Z_{0}=z_{0} / M, Z_{1}=z_{1} / M$. Therefore,

$$
y_{i} \pm X_{i}=2 A_{i} u_{i}^{2}, \quad y_{i} \mp X_{i}=2 B_{i} v_{i}^{2} \quad(i=0,1),
$$

where for $i=0,1, b=A_{i} B_{i}, z_{i}=M u_{i} v_{i}$. Solving for each $y_{i}$, substituting $y_{i}$ and $z_{i}$ into the second equation in (1.1), and simplifying gives

$$
\left(2 A_{i} u_{i}^{2}+\left(2-M^{2}\right) B_{i} v_{i}^{2}\right)^{2}-\left(M^{2}-4\right)\left(M B_{i} v_{i}^{2}\right)^{2}=4 \quad(i=0,1),
$$

and by symmetry,

$$
\left(2 B_{i} v_{i}^{2}+\left(2-M^{2}\right) A_{i} u_{i}^{2}\right)^{2}-\left(M^{2}-4\right)\left(M A_{i} u_{i}^{2}\right)^{2}=4 \quad(i=0,1) .
$$

Therefore, there are even indices $i_{0}, i_{1}, j_{0}, j_{1}$ for which

$$
\begin{array}{ll}
W_{i_{0}}=M B_{0} v_{0}^{2}, & W_{j_{0}}=M A_{0} u_{0}^{2}, \\
W_{i_{1}}=M B_{1} v_{1}^{2}, & W_{j_{1}}=M A_{1} u_{1}^{2} .
\end{array}
$$

Suppose that one of $W_{i_{0}}, W_{j_{0}}$, say $W_{i_{0}}$, does not have a primitive prime factor. By Lemma 2.4, it follows that $M=3$ and either $i_{0}=1$ or $i_{0}=6$. Since $i_{0}$ is even, we have $W_{i_{0}}=144$, from which it follows that $B_{0} v_{0}^{2}=48$. Since $B_{0}$ is squarefree, $B_{0}=3$ and $v_{0}=4$. Therefore, from the definition of the values $B_{0}$ and $v_{0}$, we deduce that $3 y_{0} \pm x_{0}=288$. If $3 y_{0}+x_{0}=288$, then it is readily verified that $y_{0}=55$ and $x_{0}=123$. In this case, $3 y_{0}-x_{0}=$ $42=2 M A_{0} u_{0}^{2}$, showing that $A_{0}=7$, and hence $b=21$. The case $c=4$, $M=3, b=21$ was checked using SIMATH, and exactly one positive integer solution exists to (1.1). If $3 y_{0}-x_{0}=288$, then it is readily verified that $y_{0}=377$ and $x_{0}=843$. In this case, $3 y_{0}+x_{0}=1974=2 M A_{0} u_{0}^{2}$, showing that $A_{0}=329$, and hence $b=987$. The case $c=4, M=3, b=987$ was also checked using SIMATH, and in this case there is exactly one solution.

We can therefore assume that $W_{i_{0}}$ and $W_{j_{0}}$ each have a primitive prime factor, say $p$ and $q$ respectively. Then by Lemma 2.3, each of $p$ and $q$ divide one of $W_{i_{1}}$ or $W_{j_{1}}$, from which it follows that each of $i_{0}$ and $j_{0}$ divide one of $i_{1}$ or $j_{1}$. Suppose that $i_{0}$ and $j_{0}$ both divide $i_{1}$. Then by the argument given in the proof of the case $c=1$, it follows that $A_{1}=1$, and hence that $W_{j_{1}}=M u_{1}^{2}$. By Lemma 2.5, it follows that $j_{1}=2$ and $u_{1}=1$. This implies that $M y_{1} \pm x_{1}=2 M$, which upon solving for $x_{1}$ and substituting into the first equation in (1.1) gives $y_{1}=M^{2}-1=W_{3}$. But $y_{0} \neq 1=W_{1}$, and so $y_{1}>y_{0} \geq W_{3}$, which is a contradiction.

We may therefore assume that $i_{0}$ divides $i_{1}$ and $j_{0}$ divides $j_{1}$. As argued in the proof of the case $c=1$, it follows that $A_{0}=A_{1}$ and $B_{0}=B_{1}$, and 
by Lemma 2.5 , this implies that $A_{0}=B_{0}=1$, hence $b=1$, which is not possible.

Assume now that $k_{0}$ and $k_{1}$ are both even. As in the previous case we obtain

$$
M^{2} y_{i}^{2}-x_{2}^{2}=4 b z_{i}^{2} \quad(i=0,1) .
$$

Since $k_{0}$ and $k_{1}$ are even, it follows that $M$ does not divide $x_{i}$, and so $\operatorname{gcd}\left(M y_{i}-x_{i}, M y_{i}+x_{i}\right)=2$. Therefore, there are integers $A_{0}, B_{0}, A_{1}, B_{1}$, $u_{0}, u_{1}, v_{0}, v_{1}$, with $z_{i}=u_{i} v_{i}$ and $b=A_{i} B_{i}$, for which

$$
M y_{i} \pm x_{i}=2 A_{i} u_{i}^{2}, \quad M y_{i} \mp x_{i}=2 B_{i} v_{i}^{2} \quad(i=0,1) .
$$

Notice that $M$ does not divide either side of these two equations. Solving for $y_{i}$, substituting $y_{i}$ and $z_{i}$ into the second equation in (1.1), and then simplifying gives

$$
\left(\left(2 A_{i}+\left(2-M^{2}\right) B_{i} v_{i}^{2}\right) / M\right)^{2}-\left(M^{2}-4\right) B_{i}^{2} v_{i}^{4}=4 \quad(i=0,1) .
$$

By symmetry, this equation can be rewritten as

$$
\left(\left(2 B_{i}+\left(2-M^{2}\right) A_{i} u_{i}^{2}\right) / M\right)^{2}-\left(M^{2}-4\right) A_{i}^{2} u_{i}^{4}=4 \quad(i=0,1) .
$$

Since $M$ does not divide $B_{i} v_{i}^{2}$ and $A_{i} u_{i}^{2}$, there are odd indices $i_{0}, j_{0}, i_{1}, j_{1}$ for which

$$
W_{i_{0}}=B_{0} v_{0}^{2}, \quad W_{j_{0}}=A_{0} u_{0}^{2}, \quad W_{i_{1}}=B_{1} v_{1}^{2}, \quad W_{j_{1}}=A_{1} u_{1}^{2} .
$$

Assume that, say $W_{i_{0}}$, has no primitive prime factor. By Lemma 2.4, the only possibility is $i_{0}=1$. It follows that $B_{0}=v_{0}=1$, and furthermore that $M y_{0}-x_{0}=2$. Solving this for $x_{0}$ and substituting it into (1.1) gives that $x_{0}=M^{2}-2=V_{2}$ and $y_{0}=M=W_{2}$. Substituting this into $M y_{0}+x_{0}$ $=2 A_{0} u_{0}^{2}$ shows that $A_{0} u_{0}^{2}=M^{2}-1$, which means precisely that $j_{0}=3$. Since $A_{0}>1$, there is a primitive prime factor, say $p$, of $W_{3}$ which divides $A_{0}$. Since $p$ divides one of $A_{1}$ or $B_{1}$, and $\operatorname{gcd}\left(W_{i_{1}}, W_{j_{1}}\right)=1$, it follows that 3 divides only one of $i_{1}$ or $j_{1}$, say $j_{1}$. Therefore, $\operatorname{gcd}\left(A_{0}, W_{i_{1}}\right)=1$, and consequently, $A_{0}=A_{1}$. Therefore, $i_{1}=1, B_{0}=B_{1}=v_{1}=1$, and it is deduced as above that $j_{1}=3$, leading to $y_{1}=y_{0}$, a contradiction.

We may therefore assume that both $W_{i_{0}}$ and $W_{j_{0}}$ have primitive prime factors. As above it follows that both $i_{0}$ and $j_{0}$ divide one of $i_{1}$ and $j_{1}$. Assume first that they both divide $i_{1}$. As argued before, it follows that $A_{1}=1$, forcing $u_{1}=1$ and $j_{1}=1$. As in the previous paragraph, this implies that $y_{1}=M$. But since $y_{1}>y_{0} \geq W+2=M$, we obtain a contradiction.

We now deal with the case $c=-4$. In this case, $x_{i}$ divides $M$, and so we obtain

$$
X_{i}^{2}-y_{i}^{2}=4 b Z_{i}^{2} \quad(i=0,1)
$$


where as before $X_{i}=x_{i} / M, Z_{i}=z_{i} / M$. Therefore, there are integers, as before, such that

$$
X_{i} \pm y_{i}=2 A_{i} u_{i}^{2}, \quad X_{i} \mp y_{i}=2 B_{i} v_{i}^{2} \quad(i=0,1) .
$$

Solving for $y_{i}$, substituting in the second equation in (1.1), and simplifying yields

$$
\left(2 A_{i} u_{i}^{2}-\left(2+M^{2}\right) B_{i} v_{i}^{2}\right)^{2}-\left(M^{2}+4\right)\left(M B_{i} v_{i}^{2}\right)^{2}=4 \quad(i=0,1),
$$

and by symmetry,

$$
\left(2 B_{i} v_{i}^{2}-\left(2+M^{2}\right) A_{i} u_{i}^{2}\right)^{2}-\left(M^{2}+4\right)\left(M A_{i} u_{i}^{2}\right)^{2}=4 \quad(i=0,1) .
$$

Therefore, there are even indices $i_{0}, i_{1}, j_{0}, j_{1}$ for which

$$
\begin{array}{ll}
W_{i_{0}}=M B_{0} v_{0}^{2}, & W_{j_{0}}=M A_{0} u_{0}^{2}, \\
W_{i_{1}}=M B_{1} v_{1}^{2}, & W_{j_{1}}=M A_{1} u_{1}^{2} .
\end{array}
$$

We will assume for the moment that $M>1$, as this case will be proved at the end. By Lemma 2.4, $W_{i_{0}}$ and $W_{j_{0}}$ each have a primitive prime factor, say $p$ and $q$ respectively. Then by Lemma 2.3, each of $p$ and $q$ divide one of $W_{i_{1}}$ or $W_{j_{1}}$, from which it follows that each of $i_{0}$ and $j_{0}$ divide one of $i_{1}$ or $j_{1}$. Suppose that $i_{0}$ and $j_{0}$ both divide $i_{1}$. Then by the argument given in the proof of the case $c=1$, it follows that $A_{1}=1$, and hence that $W_{j_{1}}=M u_{1}^{2}$. By Lemma 2.5, it follows that $j_{1}=2$ and $u_{1}=1$. This implies that $M y_{1} \pm x_{1}=2 M$, which upon solving for $x_{1}$ and substituting into the first equation in (1.1) gives $y_{1}=M^{2}+1=W_{3}$. But $y_{0} \neq 1=W_{1}$, and so $y_{1}>y_{0} \geq W_{3}$, which is a contradiction.

Thus, we deduce that, say, $i_{0}$ divides $i_{1}$, and $j_{0}$ divides $j_{1}$. It follows that $\operatorname{gcd}\left(A_{0}, B_{1}\right)=1$ and $\operatorname{gcd}\left(B_{0}, A_{1}\right)=1$. Therefore, $A_{0}$ divides $A_{1}$, and $B_{0}$ divides $B_{1}$, from which it follows that $A_{0}=A_{1}$ and $B_{0}=B_{1}$. By Lemma 2.5, it follows that $i_{0}=i_{1}$ and $j_{0}=j_{1}$, and hence that $z_{0}=z_{1}$.

In the case that $M=1$, the above argument goes through except if, say, $W_{i_{0}}$ does not have a primitive prime factor. By Lemma 2.4, this implies that $i_{0}$ is one of $2,6,12$, in which case $B_{0} v_{0}^{2}$ is one of $1,8,144$. By recalling that $2 B_{0} v_{0}^{2}=x_{0} \pm y_{0}$, where in this case, $x_{0}^{2}-5 y_{0}^{2}=-4$, we see that there are six possible pairs of $\left(x_{0}, y_{0}\right)$ to deal with; namely, those pairs of integers $\left(x_{0}, y_{0}\right)$ which satisfy $x_{0}^{2}-5 y_{0}^{2}=-4$, and for which $x_{0} \pm y_{0} \in\{2,16,288\}$. In particular,

$$
\left(x_{0}, y_{0}\right) \in\{(1,1),(3,1),(11,5),(29,13),(199,89),(521,233)\} .
$$

If $y_{0}=1$, then $b=0$, which is not possible. If $x_{0}+y_{0}=16$, then $\left(x_{0}, y_{0}\right)=$ $(11,5)$, in which case $B_{0}=2$, while $x_{0}-y_{0}=6$, forcing $A_{0}=3$, and hence $b=6$. As there is exactly one solution to (1.1) with $c=-4, M=1, b=6$ (checked with SIMATH), this case is settled. In a similar manner it is shown that the other possible values for $\left(x_{0}, y_{0}\right)$ lead to $b \in\{42,55,377\}$, and in 
each case, (1.1) with $c=-4$ and $M=1$ has precisely one solution. This completes the proof of the theorem.

Acknowledgements. The author gratefully acknowledges support from the Natural Sciences and Engineering Research Council of Canada.

\section{References}

[1] M. A. Bennett, On the number of solutions of simultaneous Pell equations, J. Reine Angew. Math. 498 (1998), 173-199.

[2] M. A. Bennett, M. Cipu, M. Mignotte and R. Okazaki, On the number of solutions of simultaneous Pell equations II, Acta Arith. 122 (2006), 407-417.

[3] Y. Bilu, G. Hanrot and P. M. Voutier, Existence of primitive divisors of Lucas and Lehmer numbers, J. Reine Angew. Math. 539 (2001), 75-122.

[4] M. Cipu and M. Mignotte, On the number of solutions to simultaneous hyperbolic Diophantine equations, J. Number Theory, to appear.

[5] J. H. E. Cohn, Eight Diophantine equations, Proc. London Math. Soc. (3) 16 (1966), $153-166$.

[6] - Five Diophantine equations, Math. Scand. 21 (1967), 61-70.

[7] S.-I. Katayama and C. Levesque, On simultaneous diophantine equations, Acta Arith. 108 (2003), 369-377.

[8] W. Ljunggren, Einige Eigenschaften der Einheiten reeller quadratischer und reinbiquadratischer Zahlkörper mit Anwendung auf die Lösung einer Klasse unbestimmter Gleichungen vierten Grades, Oslo Vid.-Akad. Skrifter 1936, no. 12, 1-73.

[9] L. J. Mordell, Diophantine Equations, Academic Press, New York, 1969.

[10] P. Ribenboim, Square classes of Fibonacci and Lucas numbers, Portugal. Math. 46 (1989), 159-175.

[11] A. Togbe, P. M. Voutier and P. G. Walsh, Solving a family of Thue equations with an application to the equation $x^{2}-D y^{4}=1$, Acta Arith. 120 (2005), 39-58.

[12] P. M. Voutier, Primitive divisors of Lucas and Lehmer sequences, Math. Comp. 64 (1995), 869-888.

[13] P. G. Walsh, A note on a theorem of Ljunggren and the Diophantine equations $x^{2}-k x y^{2}+y^{4}=1,4$, Arch. Math. (Basel) 73 (1999), 119-125.

[14] P. Z. Yuan, On the number of solutions of $x^{2}-4 m(m+1) y^{2}=y^{2}-b z^{2}=1$, Proc. Amer. Math. Soc. 132 (2004), 1561-1566.

Department of Mathematics

University of Ottawa

585 King Edward St.

Ottawa, Ontario, Canada K1N 6N5

E-mail: gwalsh@mathstat.uottawa.ca

Received on 12.2.2006

and in revised form on 26.10.2006 